\title{
Quantum transitions induced by the third cumulant of current fluctuations
}

\author{
T. Ojanen* and T. T. Heikkilä \\ Low Temperature Laboratory, Helsinki University of Technology, P. O. Box 2200, FIN-02015 HUT, Finland
}

(Dated: July 15, 2018)

\begin{abstract}
We investigate the transitions induced by non-Gaussian external fluctuations on a small quantum system. The rates for the transitions between the energy states are calculated using the real-time Keldysh formalism for the density matrix evolution. We detail the effects of the third cumulant of current fluctuations coupled to a quantum system with a discrete level spectrum and propose a setup for detecting the frequency-dependent third cumulant through the transitions it induces. We especially discuss a scheme where the fluctuations are coupled to a Josephson flux qubit.
\end{abstract}

The study of fluctuations has been in the center of interest in physics for decades. The relevance of noise and fluctuations is underlined by the fundamental relation between fluctuations and dissipation in physical systems. One very concrete example of fluctuations is the current noise in electric circuits. At equilibrium, it obeys the fluctuation-dissipation theorem which relates the magnitude of fluctuations to the temperature and the impedance of the circuit. For a quantum system with a finite number of levels interacting with an environment, the magnitude of these fluctuations in the environment then determines the steady state of the system, along with the rate with which this steady state is approached.

During the past decade, the theory of electric fluctuations in mesoscopic systems has been significantly developed to characterize them also out of equilibrium, 1.2 where a finite average current leads to shot noise. The study yields information about the microscopic physical phenomena inside electric conductors and the effects of the electromagnetic environment on mesoscopic circuits. In large wires the current statistics is Gaussian and fully characterized by the average current and the noise power. The experimental development in manufacturing smaller circuits has enabled the study of the non-Gaussian character of fluctuations in mesoscopic samples ${ }^{3.4}$ In principle, the knowledge of these fluctuations allows for an improved characterization of the conductors,$\frac{1}{.}$ or the study of the effect their non-Gaussian character causes on other mesoscopic systems 5.6 .7 .8

With a nonvanishing average current, the probability distribution of current fluctuations no longer needs to be symmetric around the average current. In particular, the third cumulant of fluctuations describing the skewness of the current distribution may be finite. It is also the lowest cumulant indicating a non-Gaussian distribution. Despite the strong theoretical effort describing the nature of the higher-order cumulants, $\frac{1}{,}$ measuring even the third cumulant with conventional techniques has turned out to be difficult and so far its only measurements exist for the case of a tunnel junction ${ }^{3}$ The attention is thus turning towards using other mesoscopic systems as fluctuation detectors. $.5,6,9,10,11$

In this Paper we analyze the transitions caused by external fluctuations on a probe quantum system. First, we present a formula correcting the Golden Rule transition rates by taking into account the next order effects that are dependent on the third cumulant. This is essential in developing generic methods for detecting non-Gaussian fluctuations. We can establish conditions imposed to suitable probes of third-cumulant induced excitations. Although we concentrate on current fluctuations, our general analysis is independent of the physical system as long as the fluctuations are linearly coupled to the probe system. To demonstrate the results, we consider a quantum two-state system (qubit) as a probe candidate and propose a setup for measuring the effects of the frequency-dependent third cumulant of current fluctuations by a Josephson flux qubit. ${ }^{12,13}$ This can be viewed as a generalization of using qubits as spectrometers of the quantum noise power, 11 a method which has already been experimentally demonstrated 14

Our starting point is the Hamiltonian

$$
H=H_{\text {ext }}+H_{s}+H_{\text {int }},
$$

where $H_{\text {ext }}$ and $H_{s}$ describe the environment where the current fluctuates and the quantum system we use as a probe for the fluctuations, respectively, and $H_{\text {int }}$ is the interaction Hamiltonian between the environment and the probe. Motivated by the case of a current-biased Josephson junction and the magnetic interaction between two circuits considered below, we study the bilinear coupling of the form $H_{\text {int }}=g \delta I \phi$. Here $\delta I$ is the current fluctuation operator acting on the environment, $\phi$ is an operator acting on the probe system and $g$ is the coupling constant of the interaction. We assume that the quantum system is described by a set of energy eigenstates $\{|n\rangle\}$ and the average current effect $g\langle I\rangle \phi$ is included in $H_{s}$. Treating $H_{\text {int }}$ as a perturbation, the Fermi Golden Rule predicts the transition rate $\Gamma_{n \rightarrow n^{\prime}}^{(2)}=\frac{2 \pi g^{2}}{\hbar^{2}}\left|\phi_{n n^{\prime}}\right|^{2} S_{\delta I}\left(\frac{E_{n}-E_{n^{\prime}}}{\hbar}\right)$ between the eigenstates of the probe system. ${ }^{11}$ The matrix element is defined as $\phi_{n n^{\prime}}=\left\langle n|\phi| n^{\prime}\right\rangle$ and the noise power $S_{\delta I}(\omega)=\frac{1}{2 \pi} \int_{-\infty}^{\infty} e^{i \omega t}\langle\delta I(t) \delta I(0)\rangle d t$. The lowest-order estimate $\Gamma^{(2)}$ is thus proportional to the second cumulant of current fluctuations. The correlator in the above expression is calculated with respect to the environment Hamiltonian $H_{\text {ext }}$ as if the probe system did not exist. Below, we correct the transition rate $\Gamma^{(2)}$ by calculating the next order contribution $\Gamma^{(3)}$, depending on the third cumulant.

We solve the density matrix evolution using the realtime Keldysh method, as outlined in Refs. 15 16, which is 
a natural formalism for studying a small subsystem in a larger environment. We are interested in the dynamics of the probe system in particular, so we study the reduced density operator $\rho(t)=\operatorname{Tr}_{\text {ext }} \rho_{\text {tot }}(t)$ where $\rho_{\text {tot }}$ is the density operator for the system and the environment. The trace goes over a complete set of environment states. The idea is to solve the temporal evolution of a diagonal element of the reduced density matrix $\rho_{n^{\prime} n^{\prime}}(t)=\left\langle n^{\prime}|\rho(t)| n^{\prime}\right\rangle$ with the initial condition $\rho_{n n}\left(t_{0}\right)=1\left(n \neq n^{\prime}\right)$. In the long-time limit $\rho_{n^{\prime} n^{\prime}}(t)$ is proportional to the total evolution time $t-t_{0}$, the coefficient being the transition rate $\Gamma_{n \rightarrow n^{\prime}}$. We calculate the rates between wellspecified states of the reduced system. Therefore, without loss of generality, we use an initial state of the form $\rho_{\text {tot }}\left(t_{0}\right)=\rho_{\text {ext }}\left(t_{0}\right) \otimes \rho\left(t_{0}\right)$, where $\rho_{\text {ext }}\left(t_{0}\right)$ describes the initial state of the environment.

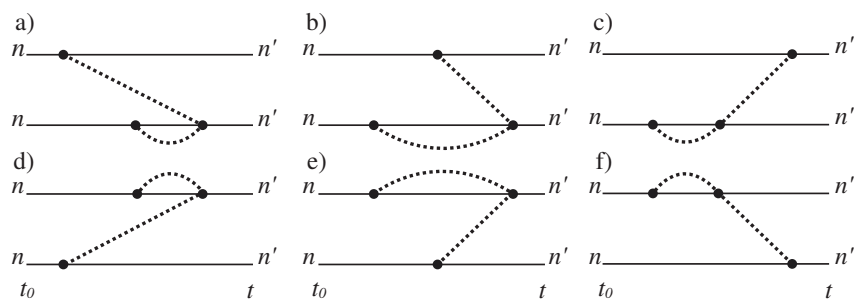

FIG. 1: Six diagrams contributing to $\Gamma_{n \rightarrow n^{\prime}}^{(3)}$. Each diagram represents temporal evolution from the initial state $|n\rangle\langle n|$ to the final state $\left|n^{\prime}\right\rangle\left\langle n^{\prime}\right|$. The dots represent interaction vertices $g \delta I \phi$ and the horizontal lines forward and backward propagation in time.

As the lowest-order contribution to $\Gamma_{n \rightarrow n^{\prime}}$ is the wellknown Golden-Rule result $\Gamma_{n \rightarrow n^{\prime}}^{(2)}$, we concentrate on the next order contribution $\Gamma_{n \rightarrow n^{\prime}}^{(3)}$. The total rate is then given by $\Gamma_{n \rightarrow n^{\prime}}=\Gamma_{n \rightarrow n^{\prime}}^{(2)}+\Gamma_{n \rightarrow n^{\prime}}^{(3)}$. Treating $H_{\mathrm{int}}=g \delta I \phi$ as a perturbation and using the graphical rules derived in Ref. 15, we find six different diagrams contributing to $\Gamma_{n \rightarrow n^{\prime}}^{(3)}$, see Fig. 11

To present the result in a compact way we define a correlator

$$
\begin{aligned}
& \delta^{3} I\left(\omega_{1}, \omega_{2}\right)=\frac{1}{(2 \pi)^{2}} \int_{-\infty}^{\infty} d\left(t_{3}-t_{1}\right) \int_{-\infty}^{\infty} d\left(t_{2}-t_{1}\right) \times \\
& \times e^{i \omega_{1}\left(t_{2}-t_{1}\right)+i \omega_{2}\left(t_{3}-t_{1}\right)}\left\langle\widetilde{T}\left[\delta I\left(t_{1}\right) \delta I\left(t_{2}\right)\right] \delta I\left(t_{3}\right)\right\rangle,
\end{aligned}
$$

where $\widetilde{T}$ denotes the anti-time-ordering operator. The time-dependent correlator is calculated with respect to the free external Hamiltonian $H_{\text {ext }}$ with the density operator $\rho_{\text {ext }}\left(t_{0}\right)$. We assume $H_{\text {ext }}$ to be independent of time and $\rho_{\text {ext }}\left(t_{0}\right)$ to describe a stationary state with respect to $H_{\text {ext }}$. Our results can be also stated with the help of the Fourier transform of $\left\langle\delta I\left(t_{3}\right) T\left[\delta I\left(t_{2}\right) \delta I\left(t_{1}\right)\right]\right\rangle$, which is the complex conjugate of the previous correlator, so it is a matter of choice which one to use. Our definition of the frequency-dependent third cumulant (2) differs from the one studied in Ref. 17, which consists of the sum of all possible Keldysh orderings. Whereas that definition is relevant in studying the evolution of the off-diagonal density matrix dynamics, transition rates cannot be obtained from that form. If the state of the environment is invariant under time reversal as usually in equilibrium at low magnetic fields, the correlator (2) vanishes.

Evaluating and summing the different contributions shown in Fig. 1] we obtain the result

$$
\begin{aligned}
\Gamma_{n \rightarrow n^{\prime}}^{(3)}=\frac{4 \pi g^{3}}{\hbar^{3}} \operatorname{Re} \sum_{n_{1}}[ & {\left[\int_{-\infty}^{\infty} \frac{\delta^{3} I\left(\frac{E}{\hbar}, \frac{E_{n^{\prime}}-E_{n}}{\hbar}\right)}{E-\left(E_{n_{1}}-E_{n^{\prime}}\right)-i \eta} d E\right.} \\
& \left.\times \phi_{n^{\prime}, n} \phi_{n_{1}, n^{\prime}} \phi_{n, n_{1}}\right] .
\end{aligned}
$$

The summation is extended over all the eigenstates of $H_{s}$ and $\eta$ denotes a positive infinitesimal quantity. With the help of the identity $\frac{1}{x-x_{0} \pm i \eta}=P \frac{1}{x-x_{0}} \mp i \pi \delta\left(x-x_{0}\right)$, where $P$ stands for a principle value integral, we can write (3) in the form

$$
\begin{aligned}
& \Gamma_{n \rightarrow n^{\prime}}^{(3)}=-\frac{4 \pi g^{3}}{\hbar^{3}} \times \operatorname{Im} \sum_{n_{1}}\left[-i P \frac{\delta^{3} I\left(\frac{E}{\hbar}, \frac{E_{n^{\prime}}-E_{n}}{\hbar}\right)}{E-\left(E_{n_{1}}-E_{n^{\prime}}\right)}\right. \\
& \left.+\pi \delta^{3} I\left(\frac{E_{n_{1}}-E_{n^{\prime}}}{\hbar}, \frac{E_{n^{\prime}}-E_{n}}{\hbar}\right)\right] \phi_{n^{\prime}, n} \phi_{n_{1}, n^{\prime}} \phi_{n, n_{1}} .
\end{aligned}
$$

Even without the knowledge of $\delta^{3} I\left(\omega_{1}, \omega_{2}\right)$, the general results (314) contain some information about the requirements made for the meter designed to detect the thirdcumulant effects. The structure of the product of the matrix elements $\phi_{n_{i} n_{j}}$ restricts the possible physical realizations used in detecting the transitions induced by the third cumulant. Generally the operator $\phi$ should either couple several states of the system, or both matrix elements $\phi_{n, n}$ and $\phi_{n, n+1}$ should be finite.

Next we turn to study the case where the probe system is a qubit. The system Hamiltonian can be written as $H_{s}=-\frac{1}{2} B_{z} \sigma_{z}-\frac{1}{2} B_{x} \sigma_{x}$ and the interaction term as $H_{\text {int }}=g \delta I \sigma_{z}$. The system Hamiltonian has the eigenstates $\left|E_{1}\right\rangle=\alpha|\uparrow\rangle+\beta|\downarrow\rangle,\left|E_{0}\right\rangle=-\beta|\uparrow\rangle+\alpha|\downarrow\rangle$ and the eigenenergies $E_{1}=\frac{1}{2} \sqrt{B_{x}^{2}+B_{z}^{2}}, E_{0}=-\frac{1}{2} \sqrt{B_{x}^{2}+B_{z}^{2}}$. The coefficients can be parametrized as $\alpha=\cos \frac{\phi}{2}$ and $\beta=\sin \frac{\phi}{2}$, where $\phi=\arctan \left(\frac{B_{x}}{B_{z}}\right)$. We denote the energy difference between the two eigenstates as $\Delta E=$ $\sqrt{B_{x}^{2}+B_{z}^{2}}$. Using the above conventions and the general result (4), we can express the corrections to the transition rates as

$$
\begin{aligned}
& \Gamma_{E_{1} \rightarrow E_{0}}^{(3)}=\frac{16 \pi g^{3}}{\hbar^{3}} F\left(\frac{\Delta E}{\hbar}\right)(\alpha \beta)^{2}\left(\alpha^{2}-\beta^{2}\right) \\
& \Gamma_{E_{0} \rightarrow E_{1}}^{(3)}=-\frac{16 \pi g^{3}}{\hbar^{3}} F\left(-\frac{\Delta E}{\hbar}\right)(\alpha \beta)^{2}\left(\alpha^{2}-\beta^{2}\right) .
\end{aligned}
$$

The function $F(\omega)$ contains the information about the third cumulant and is defined as

$$
\begin{aligned}
& F(\omega)=\operatorname{Im}\left[-i P \frac{\delta^{3} I\left(\frac{E}{\hbar},-\omega\right)}{E-\hbar \omega}+i P \frac{\delta^{3} I\left(\frac{E}{\hbar},-\omega\right)}{E}+\right. \\
& \left.+\pi \delta^{3} I(\omega,-\omega)-\pi \delta^{3} I(0,-\omega)\right] .
\end{aligned}
$$


Comparing the result (5) with the Golden Rule rates

$$
\begin{aligned}
& \Gamma_{E_{1} \rightarrow E_{0}}^{(2)}=\frac{8 \pi g^{2}}{\hbar^{2}} S_{\delta I}\left(\frac{\Delta E}{\hbar}\right)(\alpha \beta)^{2} \\
& \Gamma_{E_{0} \rightarrow E_{1}}^{(2)}=\frac{8 \pi g^{2}}{\hbar^{2}} S_{\delta I}\left(-\frac{\Delta E}{\hbar}\right)(\alpha \beta)^{2},
\end{aligned}
$$

one notices that the function $F(\omega)$ plays a similar role in $\Gamma^{(3)}$ as the noise power in $\Gamma^{(2)}$.

Supposing we can control the effective magnetic fields $B_{x}$ and $B_{z}$, we can optimize the parameters $\alpha$ and $\beta$ to produce the maximum effect from $\Gamma^{(3)}$. The absolute value of the expression $(\alpha \beta)^{2}\left(\alpha^{2}-\beta^{2}\right)$ is maximized by choosing $\alpha=0.89$ and $\beta=0.46$ or vice versa, i.e., $B_{x}=1.4 B_{z}$ or $B_{z}=1.4 B_{x}$. By changing the magnitude of $\Delta E=\sqrt{B_{x}^{2}+B_{z}^{2}}$ but keeping $B_{x} / B_{z}$ fixed, one can probe $F(\omega)$ as a function of frequency.

A physical qubit always has some intrinsic noise mechanism, in solid-state realizations produced by the electromagnetic environment, which cannot be neglected (we consider the external fluctuation circuit as an additional environment). To be measurable, the external current fluctuation effects have to be significant compared to transitions due to the intrinsic noise.

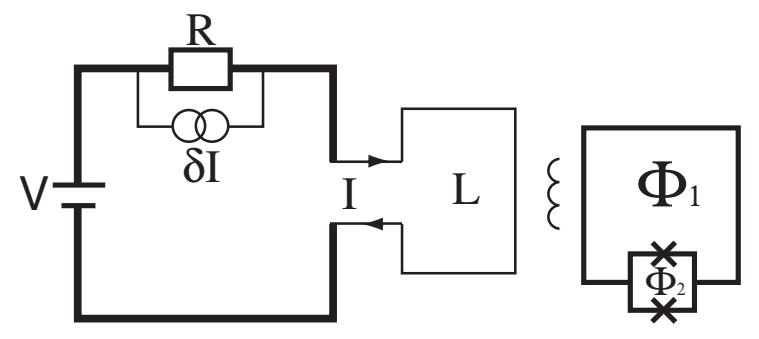

FIG. 2: Josephson flux qubit inductively coupled to an external circuit producing current fluctuations. The effective magnetic fields $B_{z}, B_{x}$ can be tuned by controlling fluxes $\Phi_{1}, \Phi_{2}$ to maximize the effects of $\Gamma^{(3)}$.

A possible physical realization for the system considered above is a Josephson flux qubit ${ }^{12,13}$ coupled inductively to the external circuit, see Fig. 20 The interaction Hamiltonian is of the form $H_{\text {int }}=\frac{M \Delta \phi}{2 L_{q b}} \delta I \sigma_{z}$, where $M$ is the mutual inductance between the qubit and the external circuit, $\Delta \phi$ is the flux difference between the two states of the flux qubit and $L_{q b}$ is the inductance of the qubit. We choose $\langle\delta I\rangle=0$, since the effects of the finite average external current can be included in redefining $B_{z}$ or eliminated by a flux control. The effective magnetic fields can be controlled by external fluxes through the loops so the qubit can be biased to the optimal point for detecting $\Gamma^{(3)}$. The transition rates follow from Eqs. (5) and (7) after the identification $g=\frac{M \Delta \phi}{2 L_{q b}}$. We assume that the energy gap to the higher states is large compared to any other energy scales in the system, allowing us to make the two-state approximation and to neglect the effective interaction terms nonlinear in $\delta I$.

Let us estimate $\Gamma^{(3)}$ in a flux qubit for a specific setup. Suppose that the external circuit consists of a scatterer with resistance $R$ and loop inductance $L$. We assume that the third cumulant of current fluctuations in the scatterer is frequency independent in the frequency scale of the circuit, $\omega_{L} \equiv R / L$. This is generally the case provided that the voltage $e V$ over and the Thouless energy $E_{T}$ of the scatterer, defined as the inverse time of flight through it, satisfy $e V, E_{T} \gg \hbar \omega_{L}: \frac{17.18}{12}$ Then the frequency dependence of the correlator (2) arises solely from the classical effect of the inductance $L$ modifying the noise. In this limit Eq. (2) can be approximated by

$$
\delta^{3} I\left(\omega_{1}, \omega_{2}\right)=\frac{F_{3} e^{2} I(2 \pi)^{-1}}{\left(1+\frac{i \omega_{1} L}{R}\right)\left(1+\frac{i \omega_{2} L}{R}\right)\left(1-\frac{i\left(\omega_{1}+\omega_{2}\right) L}{R}\right)},
$$

where $I$ is the average current in the circuit and $F_{3}$ is a scatterer-specific proportionality constant ("Fano factor") between the third cumulant and the current.

In deriving (8), we assumed $L_{q b} I_{q b}^{2} \ll L I^{2}$ where $I_{q b}$ is the current in the qubit, allowing us to neglect the backaction of the qubit on these fluctuations. This leads to the rates $\Gamma_{E_{0} \rightarrow E_{1}}^{(3)}=\Gamma_{E_{1} \rightarrow E_{0}}^{(3)} \equiv \Gamma^{(3)}$ given by

$$
\Gamma^{(3)}=A \frac{\Delta E \omega_{L}^{3}}{\left(\Delta E^{2}+\hbar^{2} \omega_{L}^{2}\right)\left(\Delta E^{2}+4 \hbar^{2} \omega_{L}^{2}\right)},
$$

where $A \equiv 32 \pi F_{3} e^{2} I g^{3}(\alpha \beta)^{2}\left(\beta^{2}-\alpha^{2}\right)$. The noise power for the setup can be written as

$$
S_{\delta I}(\omega)=\frac{F_{2}\left(e I-\frac{\hbar|\omega|}{R}\right) \theta(e V-\hbar|\omega|)+\frac{\hbar \omega \theta(\omega)}{R}}{1+\omega^{2} / \omega_{L}^{2}}
$$

where $F_{2}$ is the Fano factor for the second cumulant and $\theta(x)$ is the Heaviside step function. This formula includes the quantum fluctuations (last term) and is valid for our case provided that the temperature $T$ is low, $k_{B} T \ll \Delta E$. In the limit $e V \gg \Delta E$ we get from Eqs. (7), (9) and (10) that $\Gamma_{E_{1} \rightarrow E_{0}}^{(2)}=\Gamma_{E_{0} \rightarrow E_{1}}^{(2)} \equiv \Gamma^{(2)}$ and

$$
\gamma_{3} \equiv \frac{\Gamma^{(3)}}{\Gamma^{(2)}}=2\left(\beta^{2}-\alpha^{2}\right) \tilde{g} \frac{F_{3}}{F_{2}} \frac{\Delta E \hbar \omega_{L}}{\Delta E^{2}+4 \hbar^{2} \omega_{L}^{2}},
$$

with $\tilde{g}=\left(\frac{M \Delta \phi e}{\hbar L_{q b}}\right)$. For the optimal parameters $\alpha$ and $\beta$ mentioned above, $\alpha^{2}-\beta^{2}=0.58$. The phase difference of the two flux states can be of order $\Phi_{0} / 4=h / 8 e$, so we may estimate $\tilde{g} \approx\left(\frac{2 \pi M}{8 L_{q b}}\right)$. Consequently, it can be made of order unity or greater by an efficient inductive coupling and a large external inductance $L$. The factor $F_{3} / F_{2}$ depends solely on the nature of noise produced by the scatterer $\stackrel{19}{19}$ For realistic parameters $\omega_{L} /(2 \pi)=$ $10 \mathrm{GHz}$ and $\Delta E / h=1 \mathrm{GHz}$ the last factor is about $2.5 \%$. Optimizing the setup one could expect a relative effect $\left|\frac{\Gamma^{(3)}}{\Gamma^{(2)}}\right|$ up to roughly $10 \%$, which shows that the third cumulant effect can be significant.

Now suppose that the intrinsic relaxation of the qubit is caused by an independent zero-averaged fluctuating Gaussian field. Then the second-order rate should be 
replaced by the sum of rates caused by the field and the external circuit, the third-order rate remaining unchanged. In the case of a zero-temperature environment, this intrinsic relaxation rate $\Gamma_{\text {int }}$ can be quantified by the $Q$-factor, $\Gamma_{\text {int }}=\Delta E / \hbar Q$. In this case, its ratio to the rate $\Gamma^{(2)}$ is

$\gamma_{Q}^{\mathrm{int}} \equiv \frac{\Gamma_{\mathrm{int}}}{\Gamma_{E_{0} \rightarrow E_{1}}^{(2)}}=\frac{R}{4 R_{Q}} \frac{1}{Q \tilde{g}^{2}} \frac{1+\Delta E^{2} / \hbar \omega_{L}^{2}}{F_{2}\left(\frac{e V}{\Delta E}-1\right) \theta(e V-\Delta E)(\alpha \beta)^{2}}$.

Here $R_{Q}=h / e^{2}$.

One possibility to detect $\Gamma^{(3)}$ is to let the qubit reach the stationary state and then determine the probabilities $P_{E_{0}}$ and $P_{E_{1}}=1-P_{E_{0}}$ of the states $\left|E_{0}\right\rangle$ and $\left|E_{1}\right\rangle$. This can be achieved by repeated measurements of the qubit. From detailed balance we get

$$
p \equiv \frac{P_{E_{1}}}{P_{E_{0}}}=\frac{\Gamma_{E_{0} \rightarrow E_{1}}^{(2)}+\Gamma_{E_{0} \rightarrow E_{1}}^{(3)}}{\Gamma_{E_{1} \rightarrow E_{0}}^{(2)}+\Gamma_{E_{1} \rightarrow E_{0}}^{(3)}}=\frac{1+\gamma_{3}}{1+\gamma_{Q}+\gamma_{3}}
$$

where $\gamma_{Q}=\gamma_{Q}^{\mathrm{int}}+\gamma_{Q}^{\mathrm{c}}$ and $\gamma_{Q}^{\mathrm{c}}=\left(\Gamma_{E_{1} \rightarrow E_{0}}^{(2)}-\right.$ $\left.\Gamma_{E_{0} \rightarrow E_{1}}^{(2)}\right) / \Gamma_{E_{0} \rightarrow E_{1}}^{(2)}$. Now inverting the external current
I, $\Gamma^{(3)}$ changes sign and we get $p^{\prime} \equiv P_{E_{1}}^{\prime} / P_{E_{0}}^{\prime}=(1-$ $\left.\gamma_{3}\right) /\left(1+\gamma_{Q}-\gamma_{3}\right)$. From the above relations one can solve $\Gamma_{E_{1} \rightarrow E_{0}}^{(3)}$ and $\Gamma_{E_{0} \rightarrow E_{1}}^{(3)}$ provided that the probabilities, $\Gamma_{\text {int }}$ and $\Gamma_{i}^{(2)}$ are known. One can evaluate $\Gamma_{i}^{(2)}$ by applying Eq. (7) or the rates can be determined experimentally. From Eqs. (5) and (7) we see that when $\alpha=\beta, \Gamma^{(3)}$ vanishes but $\Gamma_{i}^{(2)}$ remains finite. By keeping $\Delta E$ fixed but setting $B_{z}=0$ it is possible to measure $\Gamma_{i}^{(2)}$ independently. Figure 3 shows the asymmetry $p-p^{\prime}$ in the change of polarization with respect to the current in the source as a function of the magnitude of the current (bias voltage $V=R I$ ).

In conclusion, we have studied the transitions induced by the third cumulant of current fluctuations on a probe quantum system. We have calculated a general formula for the transition rates and propose a scheme to measure the predicted results using a Josephson flux qubit. We have shown that the third-order transition rates are governed by the variant of the third cumulant which to our knowledge has not been studied before.

We thank Valentina Brosco, Frank Hekking and Jukka Pekola for fruitful discussions. TTH acknowledges the funding from the Academy of Finland.

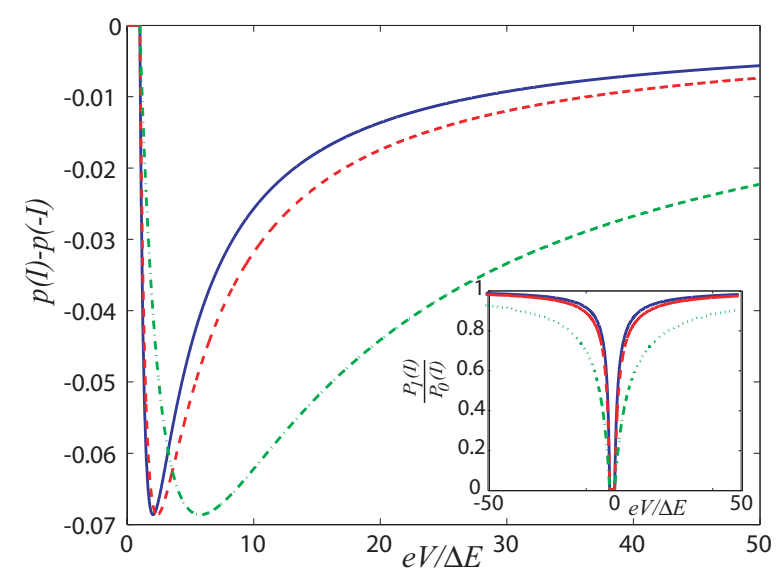

FIG. 3: (Color online) Difference $p-p^{\prime}$ in the qubit polarizations at different directions of the current for different magnitudes of intrinsic relaxation, $Q=10$ (blue solid line), $Q=1$ (red dashed line), and $Q=0.1$ (green dashdotted line). Inset shows the corresponding total polarization vs. voltage $V=R I$. Note that the behavior in the region $\mathrm{eV} \approx \Delta E$ relies on the approximations made to obtain Eq. (11). These curves have been calculated with $\alpha=0.89$, $\beta=0.46, \tilde{g}=F_{2}=F_{3}=1, \hbar \omega_{L}=2 \Delta E$ and $R=0.2 R_{Q}$.

Büttiker, Phys. Rev. Lett. 92, 176804 (2004).

19 Note that the usual results for $F_{3}$ correspond to the Keldysh symmetrized cumulant studied for example in Ref. 17. See also discussion in G. B. Lesovik and N. M. Chtchelkatchev, JETP Lett. 77, 393 (2003). 